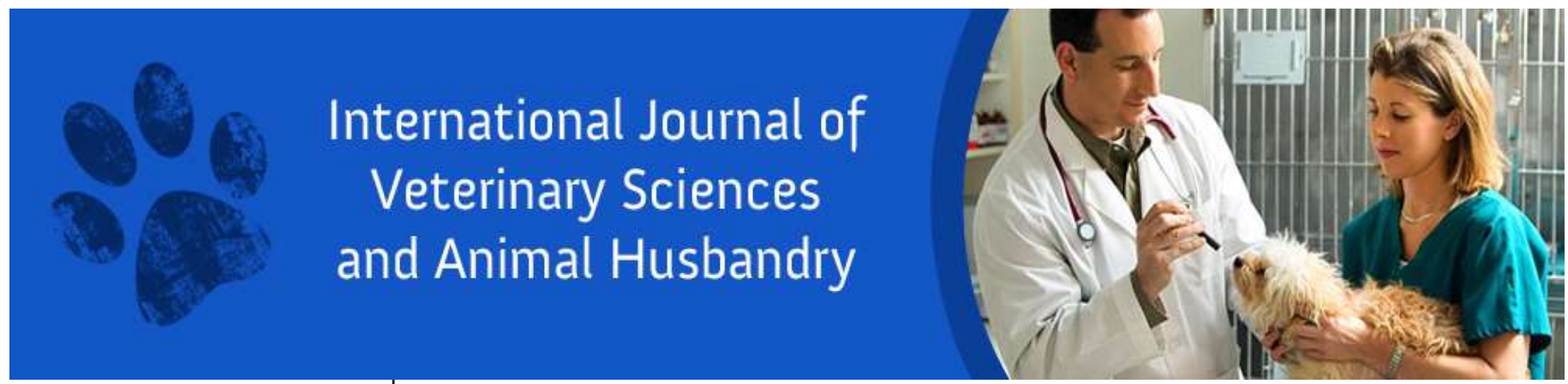

ISSN: 2456-2912

VET 2021; 6(1): 01-03 (C) 2021 VET

www.veterinarypaper.com

Received: 19-10-2020

Accepted: 02-12-2020

Dr. Vinu M Nampoothiri

Veterinary Surgeon,

Department of Veterinary

Dispensary, Morayur,

Malappuram, Kerala, India

Corresponding Author:

Dr. Vinu M Nampoothiri

Veterinary Surgeon,

Department of Veterinary

Dispensary, Morayur,

Malappuram, Kerala, India

\section{Theileriosis in cattle: Treatment and management}

\section{Dr. Vinu M Nampoothiri}

DOI: https://doi.org/10.22271/veterinary.2021.v6.i1a.314

\section{Abstract}

Bovine theileriosis is a hemoprotozoan disease transmitted by ticks. The present study was conducted at Morayur panchayath, Malappuram, Kerala by taking into consideration ten crossbred cows infected with Theileriosis. The animals had shown clinical symptoms like anorexia, anemia, pale mucus membrane, enlarged lymph node, general weakness, lameness and difficulty in getting up etc. The diagnosis was done by blood smear examination. Animals were treated with buparvaquone injection along with symptomatic and supportive therapy. Early detection and proper treatment plus management can completely cure the infection.

Keywords: Theileria, anemia, anorexia, tick, buparvaquone

\section{Introduction}

Theileria is an obligate intracellular protozoan parasite. Theileria belongs to phylum Apicomplexa, order Piroplasmida, family Theileriidae and genus Theileria. Theileria mainly affects cattle and other members of Bovidae family. Theileria species include Theileria parva which is the most important and pathogenic one causing East Coast Fever. Theileria annulata causes Tropical theileriosis or Meditterranian theileriosis. It is seen in the Mediterranean and Middle East area, from Morocco to Western parts of India and China. Theileria orientalis causes Oriental theileriosis or Theileria associated bovine anaemia (TABA). Theileria also found to be pathogenic in small ruminants. Theileria species are spread by ticks. Theileria parva is transmitted by Rhipicephalus ticks and Theileria annulata is transmitted by ticks of the genus Hyalomma. Ticks can remain infected on the pasture for up to two years. Sporozoites are transmitted to susceptible animals in the saliva of the feeding tick. Theileria mature and enter the saliva after the tick attaches to the host. Schizonts occur in spleen, lymph nodes, liver and whole blood from Theileria infected animals, transmission by inoculated schizonts occurs readily to cattle. This study describes the clinical symptoms and successful therapeutic management of the disease in cattle.

\section{History and Clinical Observations}

The present study was conducted on ten crossbred cows presented with clinical signs like anorexia, anaemia, pale mucus membrane, general weakness, tick infestation, difficulty in getting up formed the material for the study. Clinical examination hasn't revealed high fever in all animals. Some animals showed normal temperature range only. But swollen lymph nodes, pale mucus membrane, general weakness and tick infestation noticed in all animals. Certain animals showed dermatitis like cutaneous lesions and intermittent occurrence of mastitis. Thin blood smears were prepared using peripheral blood collected from the ear vein of each cattle. Blood smears were labelled and fixed using methanol. Smears were stained with Giemsa stain at 1 in 10 dilutions for 30 minutes and examined under oil immersion objective of the microscope for the presence of hem parasites. 
Table 1: Clinical Examination of the Animals

\begin{tabular}{|c|c|c|c|c|c|c|c|c|c|c|}
\hline & Cow1 & $\mathbf{2}$ & $\mathbf{3}$ & $\mathbf{4}$ & $\mathbf{5}$ & $\mathbf{6}$ & $\mathbf{7}$ & $\mathbf{8}$ & $\mathbf{9}$ & $\mathbf{1 0}$ \\
\hline Temperature & $104.4 \mathrm{~F}$ & $102.2 \mathrm{~F}$ & $101.8 \mathrm{~F}$ & $103.6 \mathrm{~F}$ & $104.2 \mathrm{~F}$ & $101.6 \mathrm{~F}$ & $102.4 \mathrm{~F}$ & $101.8 \mathrm{~F}$ & $102.2 \mathrm{~F}$ & $103.2 \mathrm{~F}$ \\
\hline Mucus Membrane & Pale & Pale & Pale & Pale & Pale & Pale & Pale & Pale & Pale & Pale \\
\hline Lymph Node & Enlarged & Enlarged & Enlarged & Enlarged & Enlarged & Enlarged & Enlarged & Enlarged & Enlarged & Enlarged \\
\hline Anorexia & Yes & Yes & No & Yes & Yes & No & Yes & No & Yes & Yes \\
\hline Anemia & Yes & Yes & Yes & Yes & Yes & Yes & Yes & Yes & Yes & Yes \\
\hline General Weakness & Yes & Yes & No & Yes & Yes & No & Yes & No & Yes & Yes \\
\hline Lameness & No & No & No & Yes & Yes & Yes & No & Yes & No & Yes \\
\hline Difficulty in getting up & No & No & No & Yes & Yes & Yes & Yes & Yes & No & Yes \\
\hline
\end{tabular}

\section{Diagnosis and treatment}

On microscopic examination Theileria could be detected on the blood smears. Theileria is a disease that causes various symptoms unlike other diseases because of its ability to cause multiple organ failure. From the history, clinical signs and blood smear the disease was confirmed as Theileriosis.

All the affected animals were treated with Inj. Zubion (Buparvaquone @ $2.5 \mathrm{mg} / \mathrm{kg} \mathrm{b}$. wt. Intramuscularly at two different sites in neck muscles on day one, Inj. Belamyl (Thiamine hydrochloride, Riboflavin, Niacinamide, Vitamin B12, Liver extract) @10 ml. intramuscularly on day one, Liq. aRBCe RAKHT (haematinic) 50ml BID for the next two weeks and Bexoliv (liver tonic) 30ml BID for the next two weeks. This was the general treatment protocol followed. In addition to these, according to the variation in clinical manifestations and general condition of the animal certain additional drugs were used. In animals with high temperature Inj. Melonex plus (Meloxicam, Paracetamol) @ $0.5 \mathrm{mg} / \mathrm{kg}$ b.wt. Intramuscularly on day one were used. Animals with lameness and difficulty in getting up were given with Inj Urimin (Sodium acid phosphate) @ 10ml per animal. Animals which were extremely anaemic are given with Inj Vetplasma (Hydroxy ethyl starch in isotonic sodium chloride intravenous infusion 6\%) @ 1000ml per animal and Inj. Dexona (Dexamethasone sodium phosphate) @10ml on day one.

These animals were again tested for Theileria after 7 days. Among the ten animals six of them again showed Theileria organisms in blood smears. They were given with a second dose of Inj Buparvaquone at the same dose rate. Again the smear was taken after 7 days. Two of the animals still had the infection which was given with a third dose of Buparvaquone. All the animals became normal after treatment.

\section{Discussion}

Clinical examination not revealed high fever in all animals. On contrary to the results Singh and Verma, (2016) [5] obtained high temperature $(104 \mathrm{~F}-106 \mathrm{~F})$ on all the infected animals. Mucus membrane was pale in all animals. Singh and Verma (2016) ${ }^{[5]}$ also obtained the same observations. Lymph node enlargement is another characteristic of haemoprotozoan disease like Theileria. Another significant feature of this disease is the occurrence of anemia. It is due to the overproduction of cytokines and reactive oxygen species (Saleh et al., 2011) ${ }^{[4]}$. Oxidative stress results in anemia (Nazifi et al., 2011) ${ }^{[3]}$. Lameness and lethargy were observed in cattle infested with Theileriosis (Devadevi et al., 2018) ${ }^{[2]}$. Tropical theileriosis were also associated with dermatological lesions. Skin nodules, haemorrhagic lesions, cutaneous gangrene, etc. were reported with the presence of schizonts in the nodular lesions (Gharbi et al., 2017) ${ }^{[6]}$. Theileria had a negative effect on milk production. It also results in intermittent infections of Mastitis. Clinical theileriosis can cause significant milk production losses in dairy cattle (Perera et al., 2014) ${ }^{[9]}$.
On the treatment side buparvaquone was found effective at a dose rate of $2.5 \mathrm{mg} / \mathrm{kg}$ bodyweight intramuscular route. The result was in agreement with Devadevi et al., (2018) ${ }^{[2]}$, Singh et al., (1993) ${ }^{[1]}$. The present study showed that in some animals multiple doses of injections are needed for recovery. In contrary to the result Devadevi et al., (2018) ${ }^{[2]}$ noticed that a single dose of buparvaquone @ $2.5 \mathrm{mg} / \mathrm{kg}$ body weight intramuscular administration showed 100 percent recovery and eliminate the Theileria organism from the circulation. But Morrison (1998) ${ }^{[8]}$ stated that cross breed cows with a single dose of Inj. Buparvaquone @ $2.5 \mathrm{mg} / \mathrm{kg}$ body weight intramuscular showed $98.8 \%$ recovery only. That shows the need of a second dose. The antioxidant potential of vitamins has been demonstrated (Singh et al. 2012) [7] and the administration of these vitamins in the present cases might have helped to ameliorate the anemia of affected animals by mitigating the Theileria induced oxidative damage to erythrocytes.

Prevention of Theileria can be done by in vitro attenuated schizontal cell culture vaccine with trade name of Rakshavac $\mathrm{T}$ manufactured by Indian Immuno logicals Ltd, Hyderabad. Prevention of tick infestation is the most important step that had to be taken for the prevention of Theileriosis. Ivermectin injection @ $0.2 \mathrm{mg} / \mathrm{kg}$ body weight subcutaneously or topical application of flumethrin 1\% pour-on preparation (Bayticol pour-on) over the vertebral column can be advised.

\section{Conclusion}

From this study it was noticed that Theileria can cause various symptoms like anorexia, general weakness, anemia, lameness etc. in cattle. Buparvaquone injection intramuscularly was found to be effective at a dose rate of $2.5 \mathrm{mg} / \mathrm{kg}$ body weight. But in some animals second and third dose of injections are needed for cure. Early detection of infection is very crucial in case of Theileria. Early detection and timely treatment results in cure.

\section{References}

1. Singh J, Gill JS, Kwatra MS, Sharma KK. Treatment of theileriosis in crossbred cattle on the Punjab. Trop. Anim. Hlth. Prod 1993;25:75-78.

2. Devadevi N, Rajkumar K, Vijayalekshmi P. Efficacy of buparvaquone in the treatment of bovine benign theileriosis. Int. J Chem. Studies 2018;6(3):3110-3112.

3. Nazifi S, Razavi SM, Kianiamin P, Rakhshandehroo E. Evaluation of erythrocyte antioxidant mechanisms: antioxidant enzymes, lipid peroxidation, and serum trace elements associated with progressive anemia in ovine malignant theileriosis. Parasitol Res 2011;109:275-281.

4. Saleh MA, Mahran OM, Al-Salahy MB. Corpuscular oxidation in newborn crossbred calves naturally infected with Theileria annulata. Vet Parasitol. 2011;182(24):193-200.

5. Verma AK, Singh SK. Control and therapeutic 
management of bovine tropical theileriosis in crossbred cattle. J Parasit Dis 2016;40(1):208-210.

6. Gharbi M, Souidi K, Boussaadoun MA, Rejeb A, Jabloun $\mathrm{S}$, Gnaoui A et al. Dermatological signs in bovine tropical theileriosis (Theileria annulata infection), A review 2017;36(3): 807-816.

7. Singh SK, Dimri U, Sharma MC, Swarup D, Kumar M, Tiwary R. Psoroptes cuniculi induced oxidative imbalance in rabbits and its alleviation by using vitamins A, D3, E, and $\mathrm{H}$ as adjunctive remedial. Trop Anim Health Prod 2012;44(1):43-48.

8. Morrison WI. The Merck Veterinary Manual. Edn. 8th, Merck and Co. Inc. Whitehouse Station NJ, USA 1998,31-33.

9. Perera PK, Gasser RB, Firestone SM, Anderson GA, Malmo J, Davis G, et al Oriental theileriosis in dairy cows causes a significant milk production loss. Parasite Vectors 2014;7:73. 